them were estimated as $0.46 \%, 0.13 \%, 0.13 \%$ respectively for the first 3 and $0.06 \%$ for the last 4 diseases.

Conclusion This is the first comprehensive prevalence study on rheumatic diseases in such a sample which may reflect Turkish population because of migration in this area from nearly all over Turkey. The study will be maintained up to 3000 subject and presented at Eular 2001 Congress as a completed study.

Supported by Research Fund of Akdeniz University.

\section{THU0254 RISK FACTORS FOR BACK PAIN IN A CROSS SECTIONAL AND IN A ONE-YEAR FOLLOW UP STUDY IN NURSES}

M Brzosko, I Fiedorowicz-Fabrycy, J Fliciñski, H Przepiera-Bêdzak, K Prajs. Rheumatology, Pomeranian Academy of Medicine, Szczecin, Poland

\subsection{6/annrheumdis-2001.798}

\section{Background}

Objectives To find risk factors for back pain in retrospective study, one year follow-up study, recurrence and first episode of back pain in one year follow-up study in nurses.

Methods The study was performed on 470 nurses of clinical hospital, 92,3\% of nurses answered a questionnaire concerning information about low back pain. $358(82,5 \%)$ have answered the questionnaire on the physical activities performed at work and $369(85,0 \%)$ were examined. $87,9 \%$ of nurses who answered the first questionnaire have taken part in follow up study. The potential relationship of the investigated 47 variables like financial status, habits, physical activity or work conditions were assessed in multivariate logistic regression model and then the role of significant variables was counted as relative risk with $95 \%$ of confidence interval in univariate logistic regression model. For statistical evaluation of the data STATA 5.0 software was used.

Results The multivariate logistic regression analysis did not show any common risk factor for back pain in cross sectional, oneyear follow up study, for the first episode or for the reoccurrence of back pain in one-year follow up study. Cycling was a risk factor for back pain in both one year follow-up study and first episode (OR 1,3) and for recurrence of back pain (OR 1,9) and the same applied to work with arms elevated on the shoulder level longer than $1 \mathrm{~min}$ an hour. These two variables were the only significant risk factors $(p<0,05)$ for the first episode of back pain in one-year follow up study. Spinal mobility ranges in different planes did not have any impact on first episode of back pain. Age, bringing up children, alcohol drinking, experiencing recurrent diarrheas, abdominal pain, discontentment with own job, limited lateral trunk flexion to the right, pushing heavy objects and both hands lifting weights were risk factors only for reoccurrence of back pain in a one-year follow up study. Bad assessment of the own health was a risk factor of back pain in cross sectional, one-year follow up study and especially for the reoccurrence of back pain in one-year follow up study.

Conclusion There are different risk factors of back pain in retrospective study, one year follow-up study, recurrence and first episode of back pain in one year follow-up study in nurses.

\section{THU0255 A NEW BONE PROGNOSTIC FACTOR IN MULTIPLE MYELOMA (MM): LEVEL SERUM CROSS-LAPS (CTX) AT DIAGNOSIS}

${ }^{1} \mathrm{~A}$ Duché, ${ }^{2} \mathrm{X}$ Leleu, ${ }^{2} \mathrm{~A}$ Duhamel, ${ }^{2} \mathrm{~V}$ Delannoy, ${ }^{2} \mathrm{~T}$ Facon, ${ }^{1} \mathrm{M}$ Brazier. ${ }^{1} \mathrm{Clinical}$ Pharmacy Laboratory, Amiens, France; ${ }^{2}$ Hematology Depmartment, CHU Lile, Lille, France

\subsection{6/annrheumdis-2001.799}

Background In MM b2 microglobuline (b2) and chromosome 13 deletion (Ch 13) have demonstrated an adverse prognostic impact on response, duration and survival. Bone turn-over marquers are studied in management and follow-up of MM.

Objectives The aim of this study was to demonstrate that a new bone resorption marquer, ie. initial serum CTX level at diagnosis, is a potential prognostic factor in MM.

Methods Between March 1990 and june 2000, $122 \mathrm{MM}$ patients were hospitalised in our department. They all have an evaluation of demographic, usual biologic parameters, and an extensive evaluation of prognostics parameters at diagnosis, including b2, Ch 13 and CTX. Serum level CTX were assessed by ELISA test. Event free survival, relapse and death were achieved during follow-up. Descriptive analysis was performed, and correlations were obtained by univariate analysis.

Results descriptive analysis showed: mean age 61 years; men $57 \%$; b2 > $3 \mathrm{mg} / \mathrm{l}, 45 \%$; Ch 13, 32,5\%; C Reacive Proteine > $6 \mathrm{mg} / \mathrm{l}, 35 \%$; stage accordind to Durie -Salmon (I, II, III) respectively $17.6,25,57.4 \%$; IgG, IgA, other Ig respectively 62,25 , $13 \%$; number of bone lesions $>6,21 \%$, relapse $59 \%$, death $42 \%$. ROC curved didn't permitted to determined ideal threshold for CTX. Median CTX level (CTX $=2470 \mathrm{pmol} / \mathrm{l})$ at diagnosis was correlated to survival and relapse and CTX level at diagnosis (dCTX) was correlated to all prognostics parameters studied in an univariate stastistical analysis. DCTX $>2470$ $\mathrm{pmol} / \mathrm{l}$ is correlated to poor survival ( $\mathrm{p}=0.0151$ ) with event free survival at 30 months descriptive analysis showed: mean age 61 years; men 57\%,; b2 > 3 mg/l, 45\%; Ch 13, 32,5\%; C Reacive Proteine $>6 \mathrm{mg} / \mathrm{l}, 35 \%$; stage accordind to Durie -Salmon (I, II, III) respectively 17.6, 25, 57.4\%; IgG, IgA, other Ig respectively $62,25,13 \%$; number of bone lesions $>6,21 \%$, relapse $59 \%$, death $42 \%$.

ROC curved didn't permitted to determined ideal threshold for CTX. Median CTX level (CTX $=2470 \mathrm{pmol} / \mathrm{l})$ at diagnosis was correlated to survival and relapse and CTX level at diagnosis (dCTX) was correlated to all prognostics parameters studied in an univariate stastistical analysis. DCTX $>2470 \mathrm{pmol} / \mathrm{l}$ is correlated to poor survival $(\mathrm{p}=0.0151$ ) with event free survival at 30 months (versus 60 months). DCTX is statistically significatively associated to sexe $(\mathrm{p}=0.0383), \mathrm{b} 2>3 \mathrm{mg} / \mathrm{l}(\mathrm{p}=$ 0.0007), Ch 13 ( $\mathrm{p}=0.0504)$, C Reactive Proteine $>6 \mathrm{mg} / \mathrm{l}(\mathrm{p}$ $=0.0348)$, stage according to Durie -Salmon $(\mathrm{p}=0.0015)$, number of bone lesions $(\mathrm{p}=0.0330)$, poor survival $(\mathrm{p}=$ $0.089)$, and relapse $(\mathrm{p}=0.0018)$.

Conclusion We demonstrate in this retrospective study that dCTX level appears as a new prognostic factor on survival and relapse. It is associated to other prognostics factors, stage according to Durie -Salmon, and high number of bone lesions. Multivariate analysis must be performed to confirm the powerful of the combination of b2, Ch 13 and dCTX, as prognostic tool to sceen patients and manage thepareutics decisions. 